\title{
UNA CRÍTICA AL MODELO SUBCONSUMISTA DE PAUL SWEEZY Y PERSPECTIVAS PARA UNA REFORMULACIÓN *
}

\author{
Nora Lustig \\ El Colegio de México
}

\section{INTRODUCCIÓN}

DENTRo de las teorías marxistas sobre la crisis del sistema capitalista se pueden distinguir dos grandes corrientes: 1) aquellas que basan su argumento en la tendencia creciente de la composición orgánica del capital; y, 2) las que sitúan el origen de las crisis en la esfera de la realización. Dentro de este último grupo destacan las teorías del subconsumo; ${ }^{1}$ los elementos fundamentales que caracterizan a las teorías - marxistas y no marxistas - del subconsumo son: por un lado, que el estado "natural" al que tiende el capitalismo -en ausencia de fuerzas contrarrestantes- es el estancamiento secular; por otro, que la causa fundamental de este estado crítico del sistema económico es el resultado de una persistente tendencia a que la demanda de bienes de consumo sea deficiente. Según sean los factores a los que se atribuye ser causa de esta tendencia, las teorías del subconsumo se dividen a su vez en dos: aquellas que consideran el centro del problema en la "sobreacumulación" o ahorro excesivo - con Malthus como uno de sus principales proponentes iniciales-; y, las que identifican a la excesiva concentración del ingreso, con el consecuente bajo poder adquisitivo de las masas trabajadoras, como la causa principal del subconsumo (esta corriente tiene sus orígenes en los escritos de Sismondi) .

Entre los autores marxistas contemporáneos, Paul Sweezy es de los más vehementes propulsores de las hipótesis subconsumistas como explicación de la crisis del capitalismo - sobre todo del capitalismo maduro. Según Sweezy, un rasgo fundamental del capitalismo es que "la acumu-

* La autora agradece a Antonio Martín del Campo sus útiles comentarios a una versión preliminar de este artículo.

I Para una síntesis de las teorías marxistas de las crisis, véase Erik Wright, "Alternative Perspectives in Marxist Theory of Accumulation and Crisis" en J. Schwartz (Comp.), A Subtle Anatomy of Capitalism, Goodyear Pub. Co., 1977.

2 Para una crítica de las teorías del subconsumo, véase M. Bleaney, Underconsumption Theories, International Pub., 1976. 
lación ${ }^{3}$ aumenta como una proporción de la plusvalía y que la inversión crece como una proporción de la acumulación. Entre tanto el consumo crece porque los capitalistas aumentan su propio consumo y desembolsan una parte de su acumulación en salarios aumentados. Pero, y éste es el punto importante, como el aumento del consumo de los capitalistas es una proporción decreciente de la plusvalía total, y como el aumento de los salarios es una proporción decreciente de la acumulación total, resulta que la tasa de crecimiento del consumo... desciende con relación a la tasa de aumento de los medios de producción...

"Si cambiamos ahora nuestro ángulo de visión y consideramos la producción como un proceso técnico natural de creación de valores de uso, vemos que debe existir una relación precisa entre la masa de medios de producción... y la producción total de artículos de consumo. Además debe existir igualmente una relación precisa entre los cambios en la existencia de medios de producción (inversión) y los cambios en la producción total de artículos de consumo... En otras palabras, la proporción de la tasa de aumento en la producción total de artículos de consumo con respecto a la tasa de aumento de los medios de producción permanece invariable...

"Podemos enunciar ahora muy brevemente la esencia de la teoría del subconsumo. Puesto que los capitalistas, que controlan la dirección de los recursos y de los fondos, actúan de tal manera que se produce un descenso constante en la proporción (tasa de aumento del consumo/tasa de aumento de los medios de producción), y puesto que la naturaleza del proceso de producción impone por lo menos una estabilidad aproximada en la producción (tasa de aumento en la producción total de artículos de consumo/tasa de aumento de los medios de producción) resulta que existe una tendencia inherente al aumento en el consumo a quedarse atrás del aumento en la producción total de artículos de consumo. Como ya se ha hecho notar, esta tendencia puede expresarse en forma de crisis o de estancamiento, o de ambos"."

Entonces, la teoría de subconsumo de Sweezy se basa en dos premisas fundamentales: una tendencia a que se destine una parte creciente de la plusvalía a la inversión - consecuencia de lo que él llama "rasgo fundamental del capitalismo"-, por un lado; y, por el otro, una especie de "coeficientes fijos" entre la producción de medios de producción y de bienes de consumo; la contradicción surge desde el momento en que la producción de bienes de consumo implicada por los "coeficientes fijos"

3 Aquí se hace referencia a la acumulación en el sentido marxista: o sea, el concepto incluye al capital constante (inversión propiamente dicha) más el capital variable (salarios). Recuérdese, además, que la inversión en el sentido marxista comprende tanto los bienes de capital como otros bienes intermedios.

4 P. Sweezy, Teoría del desarrollo capitalista, México, Fondo de Cultura Económica, pp. 201-203. 
excede a la demanda - cuyo nivel está determinado por la manera en que los capitalistas utilizan la plusvalía - ${ }^{5}$ y, la manifestación aparente de esta contradicción es en la forma de crisis, o de estancamiento, o de ambas cosas.

En la sección del libro que dedica a este tema, Sweezy menciona la necesidad de hacer una formulación cuidadosa de esta teoría; con este fin introduce un modelo algebraico en el apéndice que - según Sweezysirve como sustentación lógica de tal formulación. Sin embargo, en dicho modelo, se encontraron una serie de inconsistencias que invalidan su adjudicada función sistematizadora. Es importante aclarar, sin embargo, que las críticas del modelo formal no deben extenderse automáticamente al planteamiento teórico del texto. Como más adelante se verá, el "fracaso" del modelo formal se debe a que no incorpora uno de los rasgos fundamentales de la teoría: el impacto del subconsumo sobre la tasa de ganancia, y de ésta sobre la inversión.

En la primera parte de este trabajo se introduce la crítica a la lógica interna del modelo (precedida de una presentación de éste), donde se demuestra la inutilidad del modelo para sustentar el argumento y las conclusiones que mantiene Sweezy. A continuación se indican -en términos generales - las relaciones y mecanismos de ajuste ausentes en el modelo formal y que son indispensables para una formulación fiel al texto, y posiblemente más exitosa. El artículo culmina con una breve sección de comentarios de tipo más general sobre esta teoría, y otras, del subconsumo, con el objeto de dar algunas pautas acerca de la relevancia y potencialidad de esta corriente en la investigación teórica y empírica.

\section{Formulación ALGEBRAICA DEL MODELo ${ }^{6}$}

El objetivo que persigue Sweezy es mostrar con el rigor de la lógica que en una economía capitalista existe una tendencia a aumentar la producción de bienes de consumo por encima del crecimiento de su demanda, y que esto lleva a un estancamiento económico. Para Sweezy esta demostración se logra si se comprueba que, cuando el producto aumenta a una tasa constante o decreciente, la tasa de crecimiento de la inversión que surge de las leyes fundamentales de la acumulación capitalista -que, bajo el supuesto de "coeficientes fijos" implica una cierta can-

5 A partir de sus argumentos, el "subconsumismo" de Sweezy se podría ubicar más claramente dentro de la corriente "malthusiana" porque el centro del problema está en el proceso de acumulación capitalista, independientemente del nivel de ingreso de los trabajadores o de la distribución del mismo entre éstos y los capitalistas (ya que ésta está determinada en el proceso de acumulación).

6 Este modelo - menciona Sweezy - se basó en las ideas presentadas por Otto Bauer en su libro Zwischen $Z$ wei Weltkriegen (1936). 
tidad de bienes de consumo-, es mayor que la tasa de crecimiento de la inversión que sería necesaria dado el crecimiento en la demanda de bienes de consumo, dictado, a su vez, por las leyes fundamentales de la acumulación. ${ }^{7}$

El modelo parte de la siguiente ecuación:

$$
I=s+l+k
$$

donde $I=$ ingreso nacional neto en términos de valor; $s=$ cuenta total de los salarios (igual al consumo de los trabajadores); $l=$ parte de la plusvalía destinada al consumo; y $k=$ parte de la plusvalía destinada a incrementar el capital constante (igual a la inversión).

Se supone inicialmente que la economía tiene una tasa de crecimiento del ingreso (producto) positiva, y que los tres componentes de (1) también crecen. Si se toma a $s$ y $l$ como funciones de $k \mathrm{y}$, bajo el supuesto de que un rasgo fundamental de la economía capitalista es que una proporción creciente de la plusvalía tiende a ser acumulada y una proporción creciente de la acumulación tiende a ser invertida, tenemos: ${ }^{8}$

$$
\begin{array}{llll}
s=f(k) ; & 0<f^{\prime}(k)<1 & \text { y } & f^{\prime \prime}(k)<0 \\
l=g(k) ; & 0<g^{\prime}(k)<1 & \text { y } & g^{\prime \prime}(k)<0
\end{array}
$$

Con base en el supuesto de "coeficientes fijos" entre la producción de medios de producción y de bienes de consumo, un aumento en el consumo implica un aumento proporcional en la inversión. Entonces se tiene:

$$
c=\lambda(d s+d l)
$$

donde $\lambda$ es el factor de proporcionalidad y $c$ es la adición a los medios de producción requeridas para satisfacer el incremento en el consumo $(d s+d l)$.

La condición que garantiza "un desarrollo fácil e ininterrumpido" es, entonces, que $\frac{d c}{d t}=\frac{d k}{d t}$. Entonces es suficiente demostrar que

7 Si bien Sweezy en ninguna parte definió explícitamente qué entiende por "tendencia al estancamiento", resulta extraño que considere que una economía está en proceso de estancamiento cuando la tasa de crecimiento del producto crece a un ritmo constante $\left(\frac{d^{2} I}{d t^{2}}=0\right)$.

8 La restricción de que $f^{\prime}(k)$ y $g^{\prime}(k)$ sean menores que la unidad es innecesaria. Sería cierto en el caso en que se tomaran las derivadas con respecto a la plusvalía total. 
$\frac{d c}{d t}$ y $\frac{d k}{d t}$ tienen signo diferente para concluir que el sistema tiende al desequilibrio.9

Para demostrar que $\frac{d c}{d t}$ y $\frac{d k}{d t}$ tienen signo diferente se tomó el siguiente camino.

De (1) y (4) se tiene:

$$
\begin{aligned}
& \frac{d^{2} I}{d t^{2}}=\frac{d^{2} s}{d t^{2}}+\frac{d^{2} I}{d t^{2}}+\frac{d^{2} k}{d t^{2}} \\
& \frac{d c}{d t}=\lambda\left(\frac{d^{2} s}{d t^{2}}+\frac{d^{2} I}{d t^{2}}\right)
\end{aligned}
$$

Por lo tanto, la ecuación (6) se puede re-escribir como:

$$
\frac{d c}{d t}=\lambda\left(\frac{d^{2} I}{d t^{2}}-\frac{d^{2} k}{d t^{2}}\right)
$$

Ademấs, la segunda derivada de $\frac{d I}{d t}$ se puede escribir como:

$$
\frac{d^{2} I}{d t^{2}}=\left[f^{\prime}(k)+g^{\prime}(k)+1\right] \frac{d^{2} k}{d t^{2}}+\left[f^{\prime \prime}(k)+g^{\prime \prime}(k)\right] \frac{d k}{d t}
$$

Si se supone que el ingreso nacional crece a una tasa constante o decreciente, o sea, que $\frac{d^{2} I}{d t^{2}} \leq 0$, "entonces se sigue de (8) y de las condiciones supuestas en (2) y (3) que:" 10

$$
\frac{d^{2} I}{d t^{2}}-\frac{d^{2} k}{d t^{2}}<0
$$

De (7) y (9) tenemos que:

- Sin embargo, aun cuando $\frac{d c}{d t}$ y $\frac{d k}{d t}$ tuvieran el mismo signo sus magnitudes podrían ser distintas, y la tendencia al desequilibrio subsistiría.

10 Sweezy, op. cit., p. 209. En nuestra discusión posterior se demuestra que esta proposición no se sigue. 


$$
\frac{d c}{d t}<0
$$

Por otro lado,

$$
\frac{d k}{d t}=\frac{\frac{d I}{d t}}{f^{\prime}(k)+g^{\prime}(k)+1}>0
$$

A partir de las ecuaciones (10) y (11), Sweezy concluye que se genera una contradicción en el sistema: "Los capitalistas tienden a aumentar la tasa de inversión $\left(\frac{d k}{d t}>0\right)$ pero la forma en que dejan aumentar el consumo sólo garantiza una fase descendente de la inversión $\left(\frac{d c}{d t}<\right.$ 0 ). Por lo tanto, si la tasa de inversión aumenta en realidad, la producción total de artículos de consumo mostrará una tendencia continua a superar la demanda." 11

Como de esta conclusión se deriva el supuesto de que el ingreso aumenta a una tasa constante o decreciente, para Sweezy el subconsumo es un problema de las economías "viejas", ya que si el ingreso creciera a una tasa ascendente, $\frac{d c}{d t}$ podría ser positiva. ${ }^{12}$

\section{CRítica de la lógica Interna del MOdelo}

Aquí se demostrará que - bajo los supuestos explicitados en el modelo- es imposible concluir sobre el signo de la expresión $\frac{d c}{d t} ; \mathrm{y}$, cómo esta ambigüedad invalida las conclusiones del modelo formal de Sweezy acerca de las consecuencias "estancacionistas", causadas por una tendencia al subconsumo.

Fijemos nuestra atención en la ecuación (9); las condiciones necesarias y suficientes para que tenga signo negativo son:

a) cuando el ingreso crece a una tasa decreciente, o sea, que $\frac{d^{2} I}{d t^{2}}<0$, entonces $\frac{d^{2} k}{d t^{2}}$ debe ser igual o mayor que cero o, si es menor que cero: $\left|\frac{d^{2} k}{d t^{2}}\right|<\left|\frac{d^{2} I}{d t^{2}}\right|$

11 Ibid., p. 209.

12 Ibidem, p. 209. 
b) cuando el ingreso crece a \una tasa constante $\frac{d^{2} I}{d t^{2}}=0, \frac{d^{2} k}{d t^{2}}$ debe ser estrictamente positiva.

c) cuando el ingreso crece a una tasa ascendente, $\frac{d^{2} I}{d t^{2}}>0$, se requiere que $\frac{d^{2} k}{d t^{2}}$ sea positiva y mayor que el valor absoluto de $\frac{d^{2} I}{d t^{2}}$.

A partir de las condiciones $a$ ), b) y $c$ ), se infiere que para conocer el signo de (9), o sea de $\frac{d c}{d t}$, se requiere: por un lado, conocer el signo de $\frac{d^{2} k}{d t^{2}} \mathrm{y}$, por el otro, saber si esta última es mayor, igual o menor que $\frac{d^{2} I}{d t^{2}}$ en valor absoluto. Por el supuesto de que una proporción creciente de la plusvalía se acumula en la forma de capital constante, sabemos que $\frac{d^{2} k}{d t^{2}}$ es positiva. A continuación se examinará si es posible determinar la segunda condición, o sea si $\frac{d^{2} k}{d t^{2}}>\frac{d^{2} I}{d t^{2}}$, para el caso de $c$ bajo los supuestos del modelo; para ello se retoma la ecuación (8) del modelo que - según Sweezy - es la que permite concluir sobre el signo de $\frac{d c}{d t}$ :

$$
\frac{d^{2} I}{d t^{2}}=\left[f^{\prime}(k)+g^{\prime}(k)+1\right] \frac{d^{2} k}{d t^{2}}+\left[f^{\prime \prime}(k)+g^{\prime \prime}(k)\right] \frac{d k}{d t}
$$

Los supuestos del modelo permiten afirmar que: $0<f^{\prime}(k)<1$; $0<$ $g^{\prime}(k)<1 ; f^{\prime \prime}(k)<0 ; g^{\prime \prime}(k)<0$, y se puede concluir que:

$$
\begin{aligned}
& 1<\left[f^{\prime}(k)+g^{\prime}(k)+1\right]<3 \\
& {\left[f^{\prime \prime}(k)+g^{\prime \prime}(k)\right]<0}
\end{aligned}
$$

También, por (11) se sabe que:

$$
\frac{d k}{d t}>0
$$


(2)' y (11) implican que el segundo término de la suma de (8) sea siempre negativo.

El siguiente paso es determinar, a partir de la ecuación (8) y de los supuestos del modelo, cuál debe ser el signo de $\frac{d^{2} k}{d t^{2}}$, y la relación entre la magnitud de ésta con respecto a $\frac{d^{2} I}{d t^{2}}$, bajo las tres posibles alternativas; el producto (ingreso) crece a una tasa ascendente, constante o decreciente $\left(\frac{d^{2} I}{d t^{2}}>0 ; \frac{d^{2} I}{d t^{2}}=0 ; \frac{d^{2} I}{d t^{2}}<0\right.$; respectivamente $)$.

a) Cuando $\frac{d^{2} I}{d t^{2}}<0$ (tasa decreciente), o sea que (8) debe ser negativa, se debe cumplir lo siguiente:

i) $\frac{d^{2} k}{d t^{2}} \leq 0$; o bien:

ii) $\frac{d^{2} k}{d t^{2}}>0, \mathrm{si}\left|\left[f^{\prime}(k)+g^{\prime}(k)+1\right] \frac{d^{2} k}{d t^{2}}\right|<\mid\left[f^{\prime \prime}(k)+g^{\prime \prime}\right.$ (k) ] $\frac{d k}{d t} \mid \mathrm{y}, \frac{d^{2} k}{d t^{2}}$ puede ser mayor, igual, o menor que $\frac{d^{2} I}{d t^{2}}$, según sean las magnitudes de los demás componentes, las cuales constituyen deseconomías que los supuestos del modelo no permiten inferir. Como se dijo antes, el caso $i$ ) queda eliminado por supuesto.

b) Cuando $\frac{d^{2} I}{d t^{2}}=0$

i) $\frac{d^{2} k}{d t^{2}}>0 \mathrm{y}\left|\left[f^{\prime}(k)+g^{\prime}(k)+1\right] \frac{d^{2} k}{d t^{2}}\right|=\mid\left[f^{\prime \prime}(k)+g^{\prime \prime}(k)\right]$ $\frac{d k}{d t} \mid \mathrm{y}$, aquí en forma definitiva $\frac{d^{2} k}{d t^{2}}>\frac{d^{2} I}{d t^{2}}$ (a menos que se trate del caso trivial de que tanto $\frac{d^{2} k}{d t^{2}}$, como $\frac{d k}{d t}$ sean iguales a cero).

c) Cuando $\frac{d^{2} I}{d t^{2}}>0$

i) $\frac{d^{2} k}{d t^{2}}>0 \mathrm{y}\left|\left[f^{\prime}(k)+g^{\prime}(k)+1\right] \frac{d^{2} k}{d t^{2}}\right|>\mid\left[f^{\prime \prime}(k)+g^{\prime \prime}(k)\right]$ 
$\frac{d k}{d t} \mid \mathrm{y}$, al igual que en el primer caso, $\frac{d^{2} k}{d t^{2}}$ puede ser mayor, menor o igual que $\frac{d^{2} I}{d t^{2}}$.

De lo anterior se infiere que los supuestos del modelo no permiten determinar de manera unívoca si $\frac{d^{2} k}{d t^{2}}$ es mayor, menor o igual a $\frac{d^{2} I}{d t^{2}}$, excepto para el caso en que $\frac{d^{2} I}{d t^{2}}=0$ (si se elimina la posibilidad de una solución trivial), o sea, cuando el ingreso aumenta a una tasa constante. Entonces, no se cumple la afirmación de Sweezy en el sentido de que una tendencia al subconsumo $\left(\frac{d c}{d t}<0\right)$ está asociada al estancamiento; se vio antes que aún cuando $\frac{d c}{d t}<0, \frac{d^{2} I}{d t^{2}}$ puede tener signo positivo, si $\frac{d^{2} k}{d t^{2}}>0$ y mayor que $\frac{d^{2} I}{d t^{2}} .^{13}$

Esta revisión de la lógica del desarrollo matemático del modelo pudo resultar tediosa, pero ha permitido poner de manifiesto la inutilidad de este modelo como formulación rigurosa de la teoría del subconsumo de Sweezy. La esencia de esta teoría, recordamos, la constituye el argumento de que en una economía capitalista la oferta de bienes de consumo tiende a superar la demanda, y que este desequilibrio conduce a la economía a un estancamiento secular, o a crisis periódicas (o ambas). En nuestro desarrollo anterior demostramos que de acuerdo a la lógica del

13 Para aclarar este punto podemos introducir un ejemplo aritmético. Supongamos que:

1) $f^{\prime}(k)+g^{\prime}(k)+1=2$

2) $\frac{d^{2} k}{d t^{2}}=5$

3) $f^{\prime \prime}(k)+g^{\prime \prime}(k)=-2$

4) $\frac{d k}{d t}=3$

Al sustituir estos valores en (8), se tiene que:

$$
\frac{d^{2} I}{d t^{2}}=(2 \times 5)+[(-2) \times 3]=4
$$

Por lo tanto, $\left|\frac{d^{2} k}{d t^{2}}\right|>\left|\frac{d^{2} I}{d t^{2}}\right|, \mathrm{y}$, por (9), $\frac{d c}{d t}$ tiene signo negativo a pesar de que el ingreso aumente a una tasa creciente $\left(\frac{d^{2} I}{d t^{2}}>0\right)$. 
modelo es posible encontrar que una economía crezca a una tasa creciente (lo opuesto al estancamiento) a pesar de que "sufra" dificultades de subconsumo. ${ }^{14}$

\section{Relaciones y mecanismos de ajuste aUSENTES EN EL MODELO FORMAL}

En la sección precedente se demostró que a partir de las relaciones y supuestos del modelo, la tendencia al subconsumo es compatible de manera perfecta con un producto que aumenta de manera constante; tal conclusión contradice las intenciones del autor de demostrar que las tendencias al subconsumo son causa primordial de las crisis o del estancamiento económicos.

En esta sección se tratará de detectar el "eslabón perdido" del modelo formal de Sweezy, cuya ausencia puede explicar la frustración de los intentos del autor.

En el texto Sweezy señala:

La tarea real de una teoría del subconsumo consiste en demostrar que el capitalismo tiene una tendencia innata a ampliar la capacidad de producción de artículos de consumo más rápidamente que la demanda de dichos artículos. Para decirlo de otro modo, debe mostrarse que existe una tendencia a utilizar los recursos en una forma que tuerce la relación entre la oferta potencial y la demanda potencial de artículos de consumo. Esta tendencia puede manifestarse en uno de dos sentidos: 1) la capacidad se amplía realmente y la dificultad se hace visible sólo cuando un volumen acrecentado de artículos de consumo comienza a llegar al mercado. Habrá entonces un punto más allá del cual la oferta excede a la demanda a precios normalmente lucrativos, y al pasar de este punto la producción de artículos de consumo, o la producción de capacidad adicional, o con mayor probabilidad ambas, serán restringidas. En este caso, pues, la tendencia en cuestión se manifiesta en una crisis. $\mathrm{O}$ bien; 2) hay recursos productivos ociosos que no son utilizados para producir capacidad adicional, porque se comprende que la capacidad adicional, sería redundante en relación con la demanda de las mercancías que pudiera producir. En este caso, la tendencia no se manifiesta en una crisis, sino más bien en el estancamiento de la producción. De aquí se sigue que, si se puede verificar la tendencia al subconsumo, ella podrá servir para explicar tanto las crisis como los periodos de estancamiento. ${ }^{15}$

A partir de lo anterior se deriva que cualquier modelo "subconsumista" debe contener, por lo menos, dos rasgos fundamentales:

14 Véase pp. 162 y 163 de este documento.

15 Op. cit., p. 200. 
a) Demostrar que en el sistema de producción capitalista existe una tendencia a que la oferta de bienes de consumo crezca más velozmente que la demanda.

b) Vincular esta tendencia al desencadenamiento de las crisis o del estancamiento económico, o de ambas cosas.

El modelo de Sweezy, de hecho, no logra ninguno de estos dos objetivos; y, peor aún, su desarrollo conduce a una contradicción. Sin embargo, parecería que Sweezy se habría conformado con demostrar lo primero, dando por obvio lo segundo. El problema surge -como vimos antes-, cuando se descubre que las tendencias subconsumistas de acuerdo al modelo pueden estar presentes en economías en estado expansivo, estacionario, o de estancamiento. La pregunta de rigor es ¿en qué consiste entonces el problema de que haya tendencias subconsumistas si éstas no implican que la economía se estanque o esté en crisis?

A nuestro modo de ver esta pregunta apunta justamente a la limitación fundamental del modelo; es decir, la ausencia del segundo elemento: el nexo entre el subconsumo y el desencadenamiento de crisis o estancamiento; y, precisamente es este nexo el "eslabón perdido" en la formulación de Sweezy.

Una manera posible de salvar esta limitación es mediante la incorporación de por lo menos tres relaciones: una que vincule el exceso de oferta de bienes de consumo con la tasa de ganancia; otra que vincule la tasa de ganancia al deseo de invertir (demanda de bienes de capital); $\mathrm{y}$, por último, una ecuación que relacione la tasa de crecimiento de la inversión con la del producto. Todas éstas deben suponerse relaciones causales que operan bajo los siguientes mecanismos: la generación de excesos en la oferta de bienes de consumo (acumulación de inventarios) representa un problema de realización de la plusvalía (o ganancia) que -dado que los costos ya han sido cubiertos- implica una disminución en la tasa de ganancia. La disminución en la tasa de ganancia debe afectar de manera adversa el deseo de invertir y, por lo tanto, disminuir la tasa de inversión, disminución que eventualmente redunda en un descenso en la tasa de crecimiento del producto. ${ }^{16}$ Ante esta situación adversa para la inversión, resulta difícil mantener con solidez una de las premisas en que se sustenta el desarrollo de la teoría subconsumista que aquí presentamos: la referente al comportamiento de la fracción de la plusvalía que se destina a la inversión a medida que la acumulación avanza.

Sin embargo, en el modelo de Sweezy ninguna de estas relaciones está presente. De esta manera, en dicho modelo se excluyen una serie de ca-

16 Quedaría por establecerse si esta disminución en la tasa de crecimiento representa una crisis coyuntural causada por una disminución en la demanda agregada (al disminuir la tasa de inversión); o si se convierte en un problema de largo plazo, o sea, si el descenso en la tasa de inversión implica una reducción relativa en la capacidad productiva. 
racterísticas fundamentales del funcionamiento del sistema capitalista, perdiéndose, además, la oportunidad de convertirlo en un sustento lógico de las tesis subconsumistas.

\section{COMENTARIOS GENERALES SOBRE LAS HIPÓTESIS SUBCONSUMISTAS}

Haciendo a un lado por el momento el desafortunado desenlace del modelo introducido por Sweezy, regresemos a los supuestos fundamentales de la teoría del subconsumo que éste desarrolla para delimitar la pertinencia de una formulación más exitosa. ${ }^{17}$

Las dos premisas esenciales de esta teoría son: a) la tendencia a que la proporción de la plusvalía destinada a la inversión vaya en aumento (y la destinada al consumo, por ende, en disminución); y, b) la existencia de "coeficientes fijos" entre la producción de medios de producción y bienes de consumo. Analicemos a continuación sus implicaciones. La primera implica que la proporción del producto total destinada a la inversión va en aumento a medida que éste crece (y la proporción consumida disminuye); por otro lado, esta premisa excluye la posibilidad de una diferencia entre el ahorro y la inversión ex ante, hipótesis muy cuestionable a la luz de la teoría keynesiana. El supuesto de comportamiento subyacente aquí es que a'medida que crece el producto (ingreso) los capitalistas ahorran e invierten una proporción mayor; ${ }^{18}$ teóricamente esta última es una proposición aceptable ${ }^{19}$ sin embargo, en situaciones concretas puede no ser cierta. ${ }^{20}$

Supongamos, por el momento, que la primera premisa es cierta: es decir, a medida que aumenta el producto la proporción que se desea invertir aumenta. Aisladamente esta proposición no representa problema alguno; la dificultad nace cuando introducimos la segunda premisa, o sea, la existencia de coeficientes fijos entre la producción de bienes de capital (y otros insumos) y la de bienes de consumo. Ambas condiciones deben coexistir para que pueda surgir la contradicción; por sí sola ninguna de las dos es una amenaza al equilibrio del sistema.

La importancia fundamental del supuesto de coeficientes fijos reside en que "explica" por qué el aumento en la proporción invertida no puede representar sólo un aumento en la capacidad de producir bienes de capital y otros insumos, sin que se incremente la capacidad para producir bienes de consumo. Sin embargo, para que esta relación de propor-

17 Supuestos que son también comunes a otras formulaciones subconsumistas.

18 Recuérdese que en estas formulaciones los trabajadores consumen la totalidad de sus ingresos.

19 Keynes sugiere un comportamiento similar al considerar que la propensión marginal al consumo disminuye a medida que aumenta el ingreso.

20 La mayor parte de las estimaciones de la propensión al ahorro mediante series de tiempo indican una relación proporcional entre el ahorro y el ingreso. 
cionalidad (en el tiempo) sea cierta debe suponerse la ausencia de tres elementos: a) cambios en la tecnología que hicieran más "capitalista" o indirecto el proceso de producción; $b$ ) cambios tecnológicos que modificaran la productividad de los factores; y c) rendimientos de escala crecientes o decrecientes en la producción de bienes de consumo. Basta que cualquiera de estos últimos esté presente para que la relación de proporcionalidad desaparezca. ${ }^{21}$ Por lo tanto, en el mejor de los casos la existencia de estos "coeficientes fijos" se convierte en un caso particular de supuestos más generales, cuya validez empírica depende de las características del proceso productivo y sus alteraciones en el tiempo.

Lo anterior no pretende ser una evaluación exhaustiva ni completa de la teoría del subconsumismo en general, o la de Sweezy, en particular (sobre todo porque aquí no se pusieron bajo consideración otras formulaciones, o los desarrollos posteriores del autor). Sin embargo, a partir de esta revisión se puede llegar a algunas conclusiones. La crítica que se hizo al modelo de Sweezy deja pendiente el desarrollo de una formulación rigurosa de la teoría del subconsumo donde se demuestre, por un lado, la existencia de las tendencias subconsumistas en el desarrollo capitalista y, por otro, sus efectos "estancadores" o desequilibradores. ${ }^{22}$

21 No hay que olvidarse que aun cuando hubiera una relación proporcional entre la producción de bienes de capital y la de bienes de consumo, esta proporcionalidad se da en el tiempo y, para demostrar la presencia del fenómeno subconsumista, es necesario mostrar que para cuando aumente la oferta de bienes de consumo, la demanda habrá aumentado en menor medida.

22 En cuanto a su aplicabilidad para el caso de los paises subdesarrollados, esta vertiente subconsumista que centra el problema en la sobreacumulación no parece ser adecuada ni pertinente. En todo caso, para estos países la vertiente "sismondiana" del subconsumismo puede resultar más apropiada y fértil en cuanto a su capacidad explicativa, ya que - como se recordará-, la hipótesis central de esta corriente es que la concentración del ingreso (y la pobreza masiva de los trabajadores) restringe la ampliación del mercado interno, lo cual se convierte en un obstáculo para el crecimiento económico. 